\title{
The Structural Optimization of One-Armed Planet Carrier of Wind Turbine Gearbox Based on Lightweight Structure
}

\author{
Bo HUANG ${ }^{1, a}$, Yu-Tao YAN ${ }^{2, b}$ \\ ${ }^{1}$ School of Mechanical Engineering and Automation, Northeastern University, Shenyang 110819, \\ China \\ ${ }^{2}$ School of Mechanical Engineering and Automation, Northeastern University, Shenyang 110819, \\ China \\ ahuang_bo1990@126.com, bytyan@mail.neu.edu.cn \\ Corresponding author: Bo HUANG Yu-tao YAN
}

Keywords: One-armed planet carrier, Finite element analysis, Lightweight, Structure optimization.

\begin{abstract}
One-armed planet carrier's static stress analysis and modal analysis were accomplished by the finite element method. The deformation, stress magnitude and distribution and natural frequency of the planet carrier were obtained. Under the condition of satisfying the ultimate strength and fatigue life, the planet carrier is optimized to get a lightweight structure. The optimized results showed the optimized planet carrier increases the amount of maximum deformation of $0.512 \mathrm{um}$, maximum stress increased by $143.271 \mathrm{MPa}$ and natural frequency increased by $0.4879 \mathrm{~Hz}$. In the first mode of the modal analysis, the total distortion is reduced by $82.5 \%$, mass and volume is reduced by $37.56 \%$. But the optimization improved the performance and transmission efficiency of the planet carrier and realized the purpose of lightweight structure.
\end{abstract}

With the rapid development of wind power industry, wind power technology is becoming more and more matural. The driving box is one of the most important components in the wind turbine, installed in a narrow cabin which is dozens of meters high from the ground[1]. Planet transmission work in the driving box. Planet carrier is one of the key parts, which supporting the planet gears, bearing the input torques. The load distributions on the planet gears are deeply influenced by the planet carrier's structure, whose vibration characteristics also have a profound impact on the performance and transmission efficiency of the gears[2]. However, with the power of wind turbine increases, the larger the overall sizes are, the larger the parts size are, which is extremely harmful to lives of the cabin and the tower. So it is essential to optimize structure of the planet carrier. Redundancy of materials, volumes, and qualities should be reduced by optimization. Then the goal of lightweight will be realized. Consequently, lightweight become a main research direction.

Lightweight is a comprehensive optimization of structural design, including manufacturing technologies and materials, whose goal is reducing the quality and improving performance, prolonging life and reducing costs[3]. Now it is mainly implemented by changing the manufacturing processes and structures and materials. Structural optimization is one of the most effective ways to lightweight. In the processes of structural optimization, there are three levels, size optimization, shape optimization and topology optimization[4]. By means of the shape optimization, materials and costs are reduced while there's nothing to do with the transmission. Markable achievements have been made in the research of optimization of planet carriers, at home and abroad. The topology optimization design of the minimum structure compliance was studied by Bruyneel[5], taking bridges for an example. Dong in order to achieve the goal of lightweight, maked topology optimization of overall structure of the double-arms carrier, based on the APDL language[6]. The modal analysis and fatigue analysis of the planet carrier were carried out by David. Blunt, who studied the cause of fatigue failure of the planet carrier[7]. The key factors, affecting the planet transmission of the system, were studied by Eritenel Tugan[8]. Yuan studied on deformations and stress distributions of rocker arms in one-sided plate, optimized the structure of the planet carrier considering on maximum surplus[9]. Zhang maked a finite element analysis of a one-armed planet 
carrier and got topology optimization of the structure from a lightweight perspective[10]. A onearmed whole planet carrier and a two-armed integral planet carrier were studied separately by Wang. $\mathrm{He}$ also analyzed the stresses and strains of optimized triangular planet carrier and circular one-arm planet carrier, got influence of connecting plate thickness and input plate thickness on the intensity and stiffness of planet carriers[11]. The analysis of intensity and stiffness and the structural optimization design of the planet carrier in the wind turbine gear box were carried out, and the size of the lightweight structure of the planet carrier was obtained by Pan[12].

Planet carrier, in the wind turbine gear box, is the main research direction, static analysis and modal analysis were carried out, and the structure of planet carrier is divided into optimization regions and non-optimization regions. The redundant parts of structure was optimized, providing the basises for lightweight design and optimization of one-armed carrier.

\section{Finite Element Analysis}

The cantilevered distribution of one-armed integral carrier easily lead to phenomenon, uneven forces on the planet carrier. Due to its simple structure and convenient manufacture, it is still widely used. In this paper, a $1 \mathrm{Mw}$ one-armed planet carrier is the research object. The finite element method was adopted to analyze the one-armed planet carrier. Rocker arms and planet shafts are as an integral structure. The planet carrier three-dimensional model is established in UG and then is imported into the ANSYS, shown in figure 1.

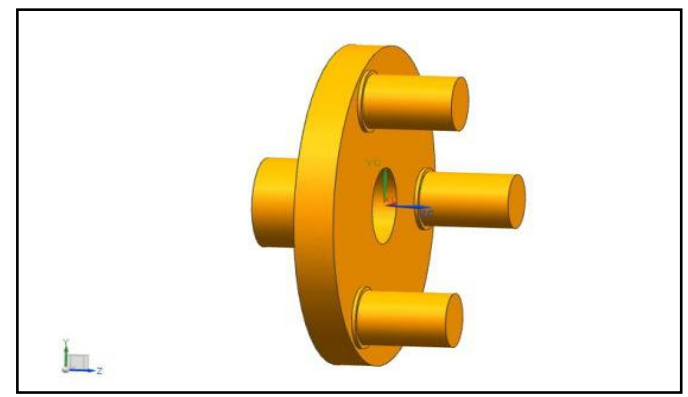

Figure 1. Structure of planet carrier

\section{Unit and Material Type Definition}

Solid185 unit is selected for the advantages of super elasticity, stress tempering, creep, large deformation and large strain capacity and so on[9]. According to the analysis of the requirements, Solid1 85 unit defined by 8 nodes, 3 degrees of freedom along the XYZ directions in each node. The detail of grid divisions has a profound influence on the results. The more detailed the grids are, the longer time and the higher the performance requirements to the computer. The size of planet carrier is so large that it is important to select the suitable size of grids. In this paper, there are 595685 elements and 111408 nodes after meshing, the length of each element is $10 \mathrm{~mm}$, as shown in Figure 2. Material parameters of one-armed integral planet carrier are shown in table 1.

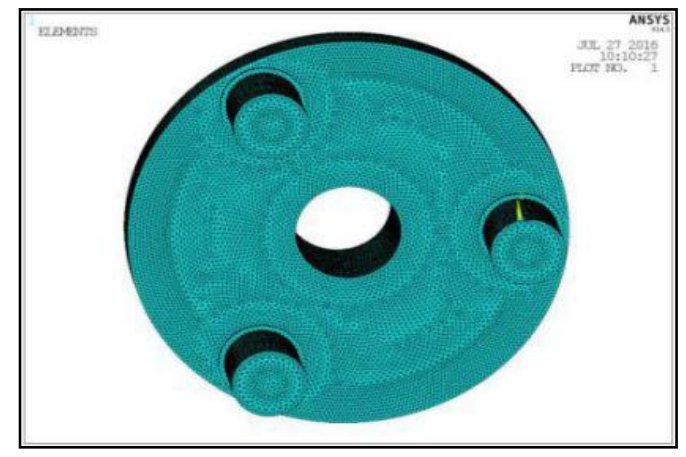

Figure 2. Meshing 
Table 1. The material parameters table

\begin{tabular}{|l|l|}
\hline Materials & $20 \mathrm{CrMnTi}$ \\
\hline Poisson's ratio $\boldsymbol{u}$ & 0.29 \\
\hline Elastic modulus $\boldsymbol{E}(\mathbf{M P a})$ & $2.07 \mathrm{e} 5$ \\
\hline Density $\boldsymbol{\rho}\left(\mathbf{k g} / \mathbf{m}^{\mathbf{3}}\right)$ & $7.8 \mathrm{e} 3$ \\
\hline Bending and fatigue strength $\boldsymbol{\sigma}_{-\mathbf{1}}(\mathbf{M P a})$ & 525 \\
\hline Torsional fatigue strength limit $\boldsymbol{\tau}_{\mathbf{- 1}}(\mathbf{M P a})$ & 300 \\
\hline Tensile mild limit $\boldsymbol{\sigma}_{\mathbf{b}}(\mathbf{M P a})$ & 1100 \\
\hline Yield strength $\boldsymbol{\sigma}_{\mathbf{s}}(\mathbf{M P a})$ & 850 \\
\hline
\end{tabular}

\section{Applied Load and Boundary Conditions}

One-armed integral planet carrier, bearing to the input torques, transmit torques to planet gears. There is a spline hole in the middle of carrier, connected with the input main shaft. During analysis, constraints and cylindrical coordinate system were set up. The circumferential constraint was set on the hole, $\mathrm{UX}=0$, the axial constraint on the head face, $\mathrm{UZ}=0$, the radial constraint at where bears supported, UY=0. Because the shape of center spline hole has nothing to do with analysis results, the spline hole was simplified as cylindrical. Three planet gears were respectively installed on the planet carrier. Whose radial forces, transmit to planet carrier, is $0 \mathrm{~N}$. So only the tangential forces transmit to the planet carrier, is $395.70 \mathrm{~N}$, whose equivalent force.

\section{Results}

The deformation nephogram of the one-armed integral planet carrier was shown in Figure 3 . The maximum deformation is $4.38 \times 10^{-5} \mathrm{~m}$, in its outermost layer. The maximum stress occurred at the root of the planet shafts, $114.365 \mathrm{MPa}$, shown in Figure 4. Its natural frequency is $0.0108 \mathrm{~Hz}$, in Figure 5. It's found that there are some redundancies in the planet carrier from the Figure 3 and Figure 4. The redundant structures should be optimized to achieve the goal of lightweight.
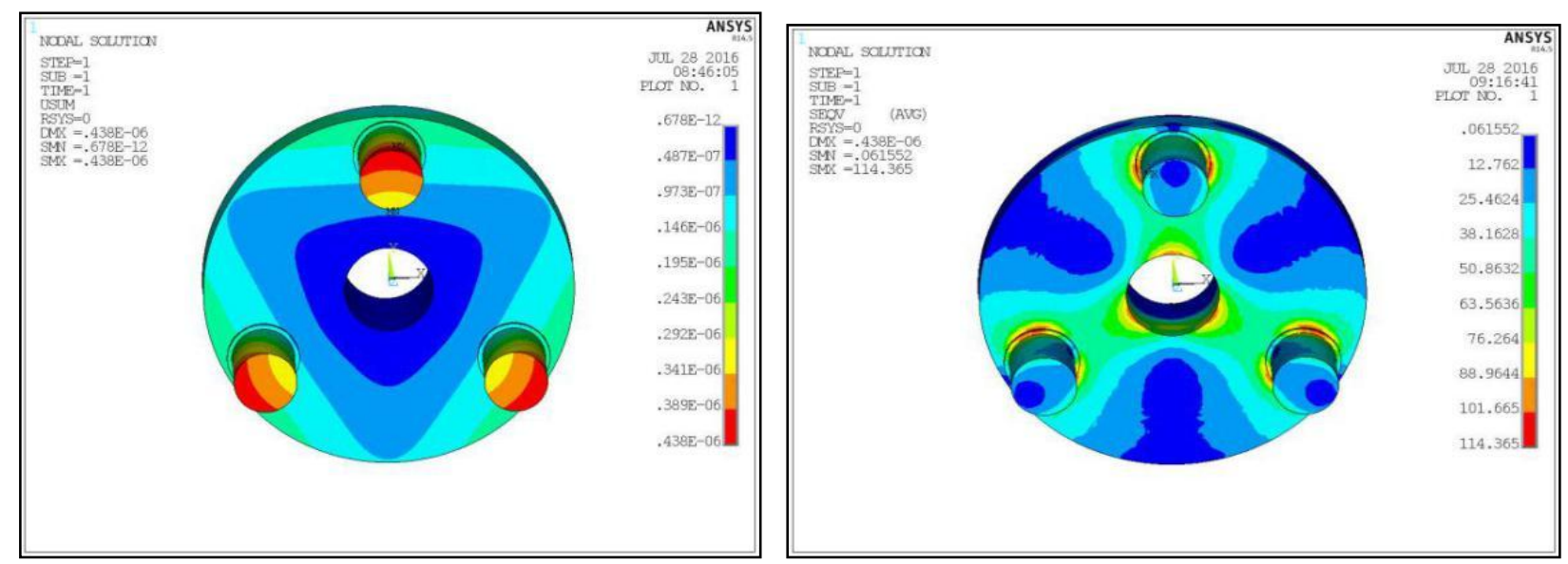

Figure 3. Deformation nephogram of planet carrier Figure 4. Stress nephogram of the planet carrier 


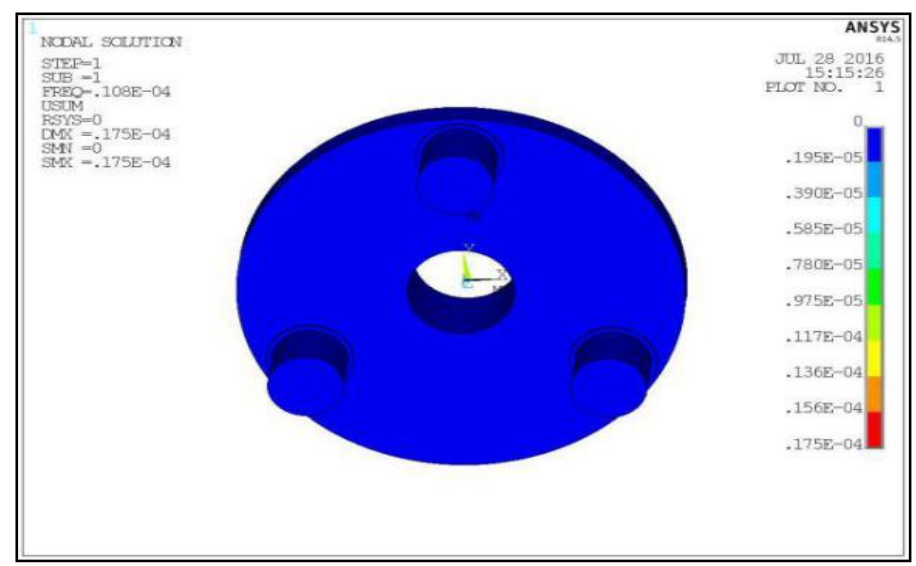

Figure 5. Natural frequence of the planet carrier

\section{Structural Optimization}

\section{Structural Optimization}

Satisfying with ultimate strength, the planet carrier of deceased volumes and reduced qualities, is the goal of structure optimization. In this paper, the structure optimization and finite element analysis were both in use.

The deformations are relative small around the hole of the planet carrier. And deformations and stresses of connecting plates, among three planet shafts, are also small, as shown in Figure 3 and 4. So they are suitable to be optimized to save materials and volumes, under the conditions of ensuring the yield strength and fatigue life. The connecting plates' between width is optimized to be $170 \mathrm{~mm}$, among the hole and planet shafts. There is an optimized circular with diameters of $610 \mathrm{~mm}$. Fillets in the connecting plates, between the hole and planet shafts, should be paid attention to avoid stress concentration. The Fillets radius is $50 \mathrm{~mm}$. At the same time, the redundancy in the connecting parts can be removed, planet carrier optimized to be structure of triangles, as is shown in Figure 6(a) and (b). Keeping applied stress loads and boundary conditions the same as before. A comprehensive comparative analysis of deformations, stresses, natural frequencies, volumes and qualities was carry out before and after.

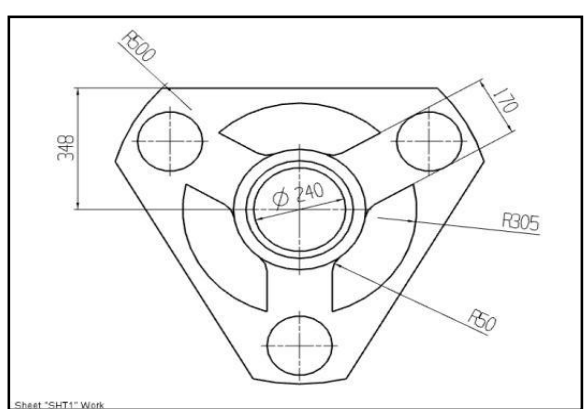

(a)Two-dimensional chart

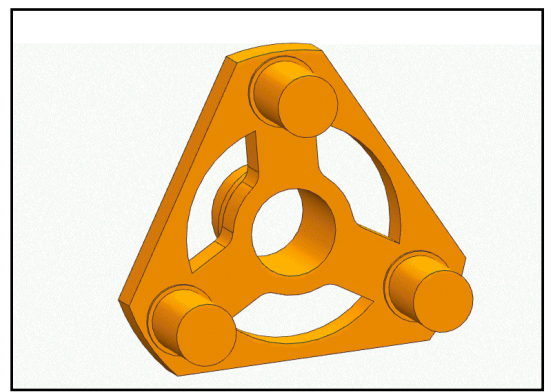

(b)Three-dimensional model

Figure 6. Structure of optimization

\section{Structural Optimization Performance Analysis}

\section{Stress and Strain}

After optimization of the one-armed integral planet carrier, the maximum deformation is $9.50 \times 10^{-}$ ${ }^{4} \mathrm{~m}$, in the outmost planet shaft, as shown in strain diagram Figure 7. This is because planet shafts are both transmitted the input torques and imposed to constraints from bears. Which lead to the biggest deformation at the end of shafts. The position of maximum deformation is the same as before, deformations were increased, reaching $116.89 \%$ (see Figure 3). The increase is only 0.512 um meeting the design requirements. Through strengthen of the structure, the one-armed 
planet carrier can be fully used. The total volume of optimized planet carrier is $5.911 \times 10^{-2} \mathrm{~m}^{3}$, reduced by $37.56 \%$, compared to the total volume of $9.446 \times 10^{-2} \mathrm{~m}^{3}$ of natural planet carrier. Through structural optimization, the volume and mass of the planet carrier were significantly reduced. And the goal of the lightweight of the planet carrier was achieved.

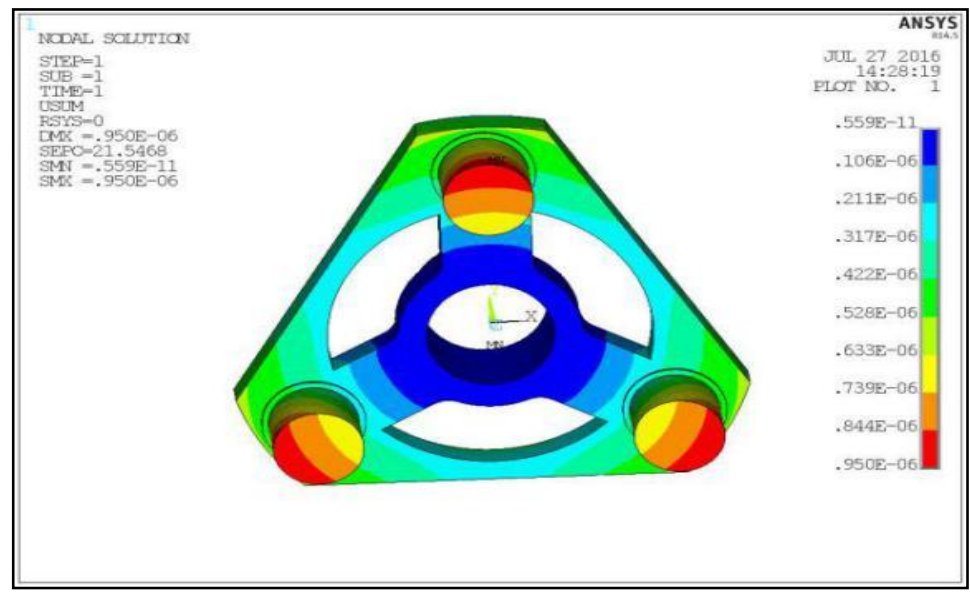

Figure 7. Deformation nephogram of optimized planet carrier

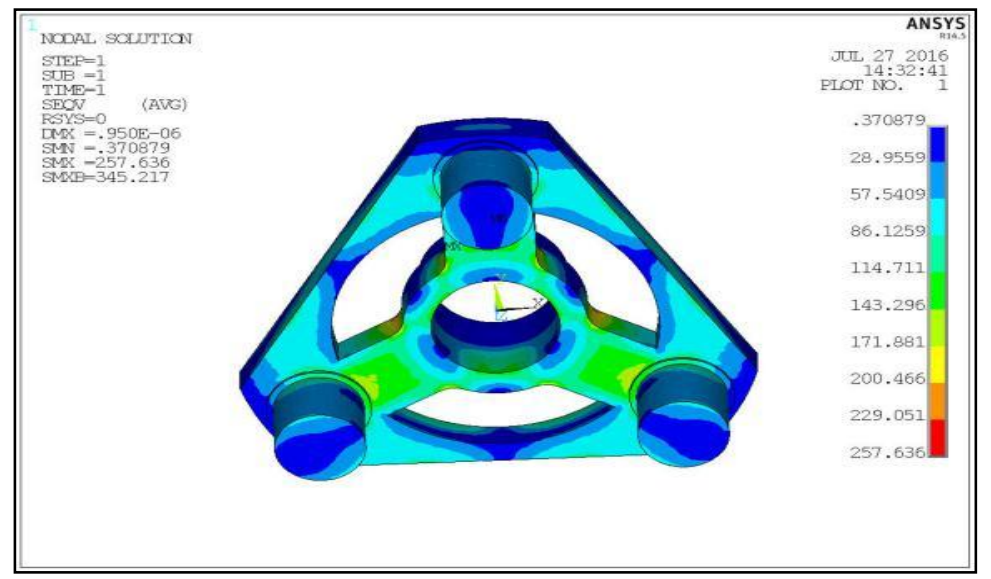

Figure 8. Stress nephogram of the optimized planet carrier

\section{Modal Analysis}

Dynamic characteristic is an another important factor, which need to take into considering. It has important influence on the stability, accuracy and efficiency of the transmission. It is easily caused by resonance that the drive failure and reduction of life span of the planet carrier. The natural frequencies and vibration modes can be determined by modal analysis. Which can avoid the resonance or the vibration of the planet at a particular frequency through the structural design.

According to the characteristics of vibration dynamics of finite element models, the differential equation of vibration is as follows.

$$
[M]\{\ddot{X}\}+[C]\{\ddot{X}\}+[K]\{X\}=\{f(t)\}
$$

$\{X\}=\left\{x_{1}, x_{2} \cdot x_{3} \ldots, x_{n}\right\}^{T} ;$

$\{f(t)\}$ - excitation force vector of structure;

$[M],[C],[K]$-total mass matrix, damping matrix, and stiffness matrix;

$\{\ddot{X}\},\{\dot{X}\},\{X\}$-Angular velocity vector, velocity vector and displacement.

If no external forces, the free vibration equation of the system is $\{f(t)\}=0$. When solving the natural frequencies and modes of free vibration, the damping effect is a little. We take not the effect 
of the damping term into consideration. Then the motion equation of the free vibration without damping is as shown.

$$
[M]\{\ddot{X}\}+[K]\{\dot{X}\}=0
$$

The characteristic equation

$$
\left\{[K]-\varpi^{2}[M]\{X\}=\{0\}\right.
$$

$\varpi$-Inherent frequency of the system. The natural frequencies and modes of the system can be get by calculating the formula (3). At the same time, the vibration mode of the planet carrier is obtained.

The inherent frequency of the planet carrier is $487.927 \mathrm{~Hz}$, shown in Figure 9. Compared to natural planet carrier, the natural frequency of the optimized planet carrier was increased. The rotation speed of planet carrier $n_{2}$ is $28.571 \mathrm{r} / \mathrm{min}$, of the planet gears $n_{H}$ is $47.618 \mathrm{r} / \mathrm{min}$, the number of teeth is 47 , the meshing frequency as follows.

$$
f=\left(\mathrm{n}_{2}-n_{\mathrm{H}}\right) \times \frac{\mathrm{z}}{60}=14.90 \mathrm{~Hz}
$$

It is known that, although the optimized structure increases the natural frequency, it still avoids the meshing frequency. So the optimized carrier planet will not produce resonance.

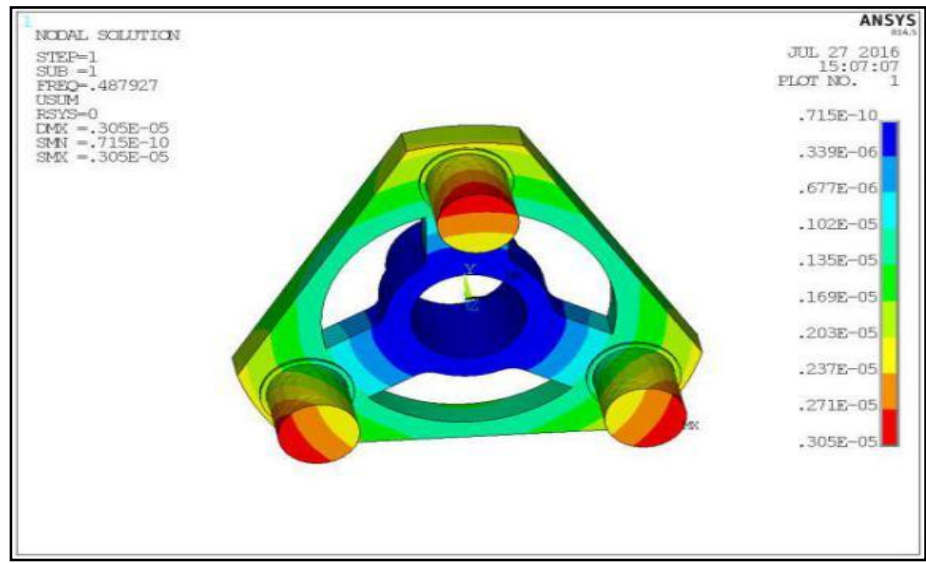

Figure 9. The natural frequence of optimized carrier planet

Deformation nephograms of modal analysis, which of the original planet carrier and optimized one, are as shown in Figure 10 and figure 11. In Figure 10, vibration modes of the carrier include the torsional vibration and shimmy. Under the operation conditions, planet carrier rotating around the center, torsional deformation had little effect on the drive performance. But the meshing of planet gears, the effect of average loads, the transmission efficiency of the one-armed integral planet carrier system, are largely affected by the deformation of the swing vibration. Compared to the original planet carrier, the total deformation is reduced by $1.44 \times 10^{-5} \mathrm{~m}$, the torsional deformation reduced by $0.36 \times 10^{-5} \mathrm{~m}$, as shown in Figure $10(\mathrm{c})$ and $11(\mathrm{c})$.

The deformations of wobble reduced by $0.1303 \times 10^{-5} \mathrm{~m}$, are shown in Figure $10(\mathrm{~d})$ and Figure 11(d). The optimized planet carrier improves the load shared performance and transmission efficiency. Which indicates the optimization of structure is reasonable and feasible. 


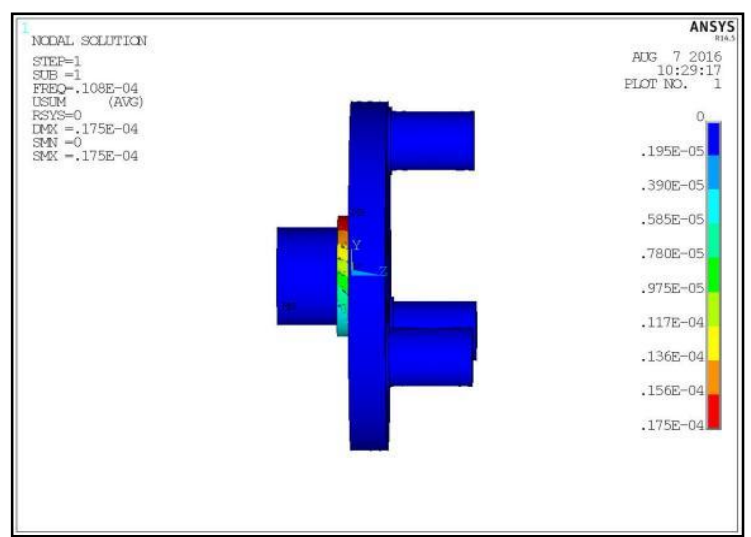

(a) Total deformation nephogram

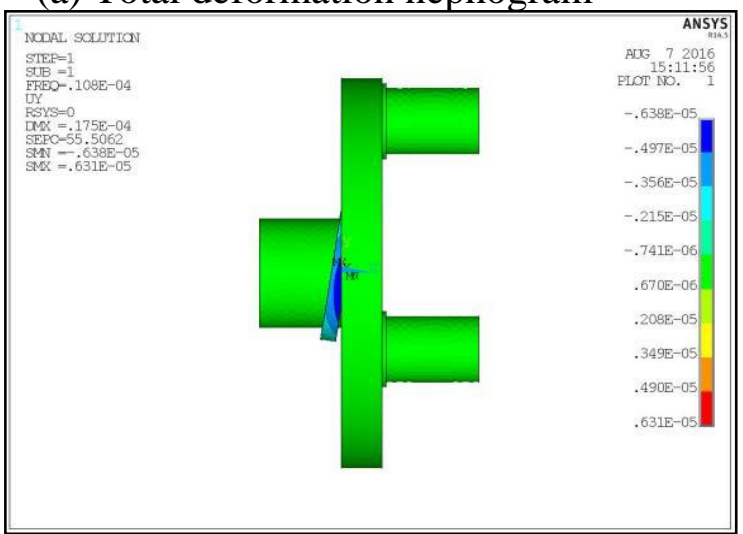

(c) Deformation nephogram along y-axis

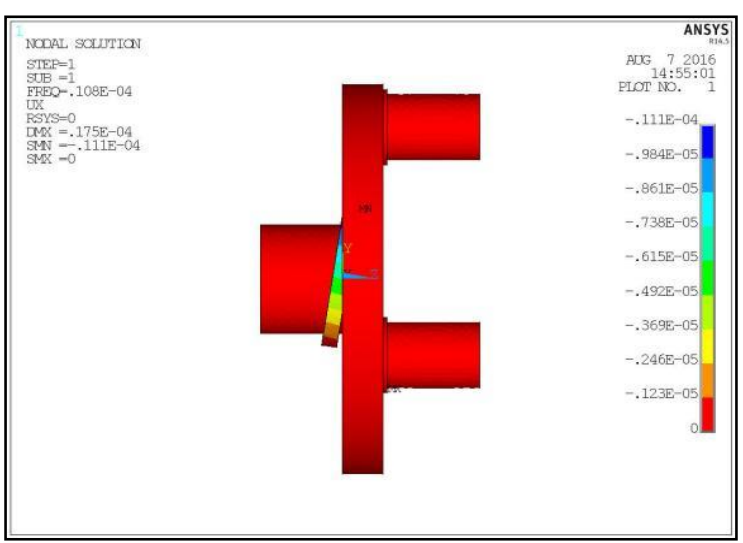

(b) Deformation nephogram along $\mathrm{x}$-axis

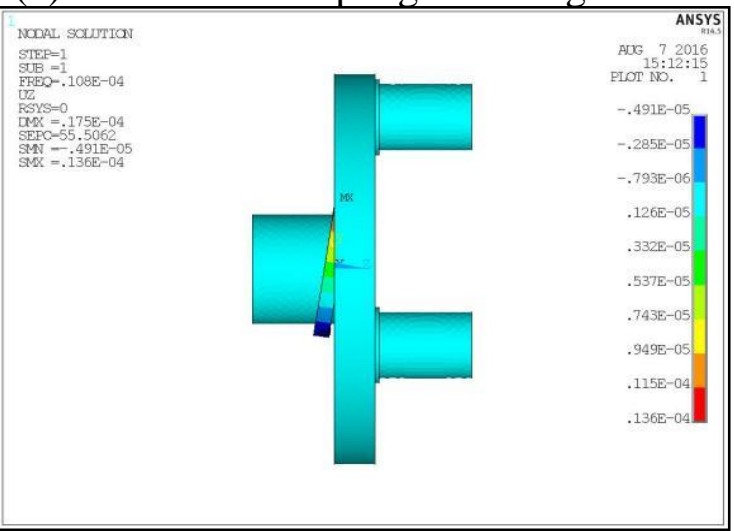

(d) Deformation nephogram along z-axis

Figure 10.The planet carrier deformation nephogram first mode

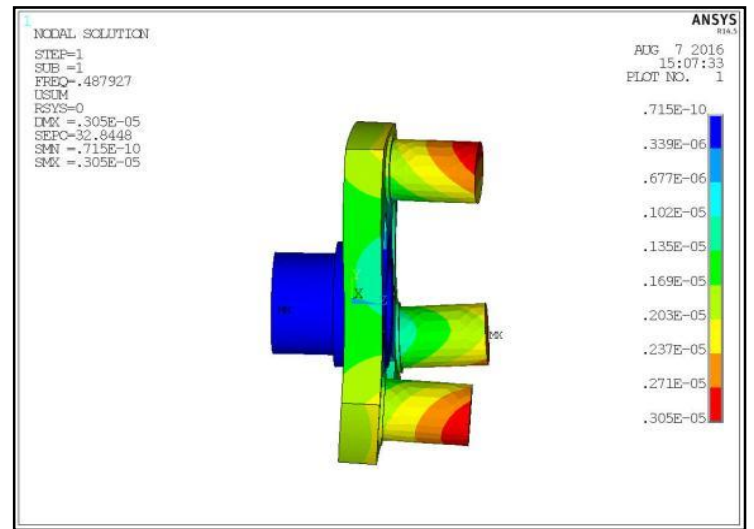

(a)Total deformation nephogram

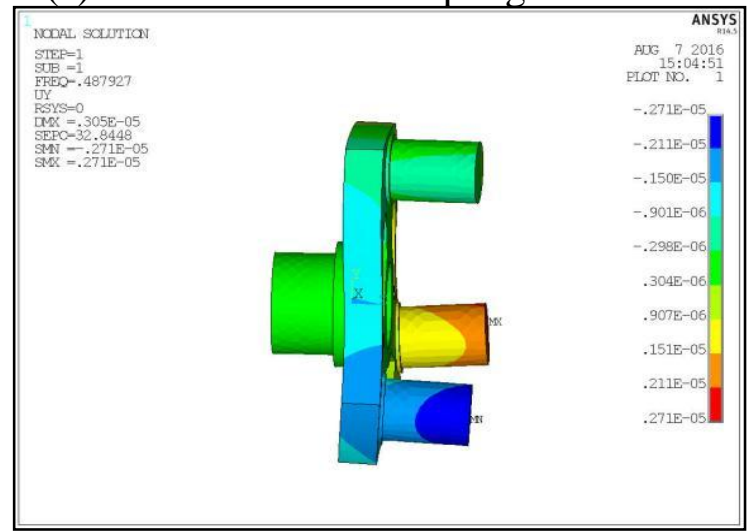

(c) Deformation nephogram along y-axis

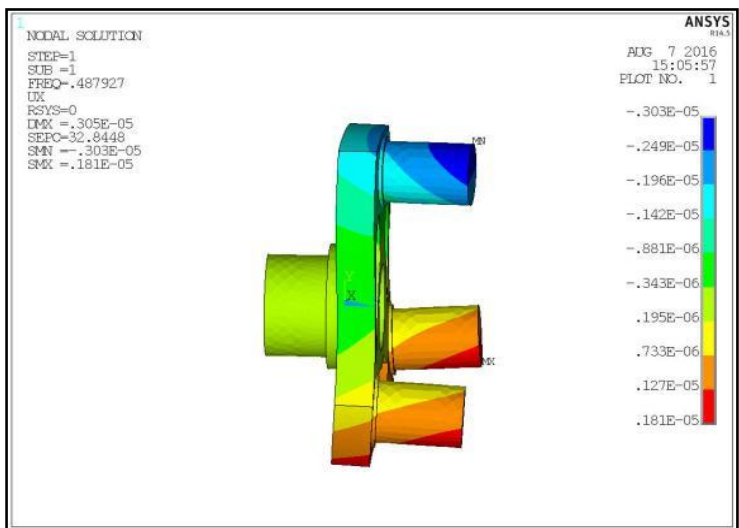

(b) Deformation nephogram along $\mathrm{x}$-axis

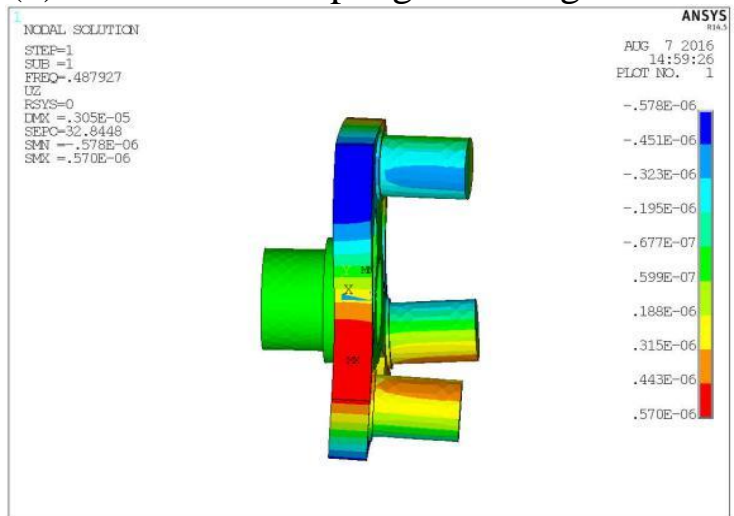

(d) Deformation nephogramalong z-axis

Figure 11. Deformation nephogram of the first mode of optimized planet carrier 


\section{Summary}

(1)Finite element analysis of one-armed integral planet carrier was carried out, the maximum deformation occurred in the outermost of planet shaft, and the stresses evenly distributed around the planet shafts. The natural frequencies, the total volumes, total qualities can be obtained.

(2)According to the analysis results, in view of lightweight, the connecting plates near planet shafts were confirmed to be optimization of region and to be optimized.

(3)The comparation between the deformations and stress distributions of the planet carrier is maked, before and after optimized. It is found that stresses and deformations are increased slightly. But the increases are much smaller than the yield strength, with satisfying the strength limit and life condition. Compared with the natural frequency, it is found that the inherent frequency was increased. But deformations of the torsional vibration and the swing vibration, affected transmission performance, are greatly reduced. And the inherent frequency of the optimized planet carrier avoid the meshing frequency. Which will not cause resonance. At the same time, the volume and mass of the optimized planet carrier, is obviously decreased, reaching $37.56 \%$. Conclusions show that structural optimization has achieved the goal of lightweight.

\section{References}

[1] Z.H. Zhang, H.P. Zhang. JOURNAL of MACHINE DESIGN, 25 (9): $54-58$ (2008).

[2] R.L. Yu. Hoisting and Conveying Machinery 7: 32-33 (2002).

[3] S.Y. He, X. Wu, W. Song. Journal of Mechanical Transmission, 2: 1-3 (2012).

[4] F. Li, D.S. Ling. Journal of Zhejiang University of Technology, 28 (3): 220-223 (2000).

[5] M. Bruyneel, P. Duysinx. Structural and Multidisciplinary Optimization, 29 (4): 245-256 (2005).

[6] H.M. Dong, Q.L. Hou, D.L. Wang. Journal of Mechanical Transmission, 37 (11): 48-66 (2013).

[7] D.M. Blunt, J.A. Keller. Mechanical Systems and Signal Procession, 7 (20): 2095-2111 (2006).

[8] T. Eritenel, R.G. Parker. Journal of Sound and Vibration, 325: 397- 420 (2009).

[9] Q. Yuan, Y. Wang. Journal of Mechanical Transmission, 34 (3): 29-32 (2010).

[10] Z.H. Zhang, Z.M. Liu, H.P. Zhang. Journal of Mechanical Transmission, 36 (6): 68-70 (2012).

[11] Z.B. Wang, Z.M. Liu. Journal of Mechanical Transmission, 38 (10): 18-20 (2014).

[12] J.K. Pan, F. Geng. Journal of Mechanical Transmission, 39 (10): 129-132 (2015). 